\title{
Programs to Recognize and Convert the Original Data of GPR*
}

\author{
Yongqing Yang \\ College of Civil and Transportation Engineering \\ Hohai University \\ Nanjing, China \\ e-mail address: yq_yang2002@hhu.edu.cn
}

\author{
Ruihu Zhu, Zhiyi Lei \\ College of Harbour, Coastal and Offshore Engineering \\ Hohai University \\ Nanjing, China
}

\begin{abstract}
Based on the description of ground penetrating radar (GPR) data processing and interpretation, this programs solve the recognition and conversion of GPR original data and display the GPR field data. The programs eliminate the built-in post-processing software reliance on software dongle. The conversion of the GPR original data turns out to be correct and reliable by means of two group different radar systems. This conversion program is more convenient to the processing and analysis of the GPR original data. GPR users can freely add some latest processing methods and modify it. The programs also provide a basic platform for the secondary development of GPR interpretation system.
\end{abstract}

Keywords-GPR; original data; recognition; conversion program; display

\section{INTRODUCTION}

Ground penetrating radar (GPR) is a highly efficient shallow geophysical detection technology. By transmitting high-frequency electromagnetic pulse wave, GPR method uses the difference of the electrical parameters of underground medium according to the kinematics and dynamics characteristics such as the amplitude, waveform and frequency of the echo to analyze and infer the medium structure and physical characteristics ${ }^{[1][2][3]}$. Comparing with the traditional geophysical methods, because of the prominent advantages of nondestructive, fast, simple and high precision's, leads to its wide attention in engineering geophysical exploration. GPR instrument system can be divided into time domain and frequency domain system. Time domain GPR system is widely used in engineering investigation. Its main advantage is radar image can intuitively reflect the structure of the target in the subsurface. Frequency domain GPR system is mainly acquiring electromagnetic wave frequency domain response of the medium by using Fourier transformation. Stepped frequency GPR system requires complex hardware system and signal processing technology ${ }^{[4][5]}$, so its development is slow, but it has better resolution and accuracy. Now GPR is applied in many fields such as geology, engineering, resource, environment, military and so on ${ }^{[6][7][8]}$.

For shallow geological survey, GPR has a greatly wide application prospect ${ }^{[7] 88^{8}[9]}$. At present, most of GPR in China are given priority to using foreign brands. But these GPR systems are limited to the software dongle . GPR users can't apply the software and modify the algorithms. So it is very inconvenient to GPR users.

\section{THEORY AND METHOD}

GPR data processing ${ }^{[10][11][12]}$ play an important role in improving the quality and interpretation of GPR profile. For GPR users working in the field of the practical production unit, they want to have a kind of simple and intuitive practical software to improve work efficiency. Now the development of GPR processing software is basically including frequencydomain filtering, frequency-wave number-domain filtering, deconvolution and migration.

GPR data processing objectives are to suppress random and rule interference. GPR image shows available reflection with the greatest possible resolution. Parameters extraction of reflected wave (electromagnetic wave velocity, amplitude and waveform) is helpful to geological interpretation. Common methods for radar data processing include digital filtering, inverse filtering, migration diffracting and enhancement processing ${ }^{[13][14]}$. Digital filtering is to use different spectrum characteristics to suppress interference wave. Inverse filtering is to obtain reflection coefficients of each layer medium interface. Migration diffracting is moving each reflection point to its original position in radar record and reflects real distribution of underground medium. GPR ' $\mathrm{CT}$ ' means a tomography technique ${ }^{[15][16]}$ that returns model parameters such as velocity, it can obtain clear image in object. It is Enhancement processing is to enhance effective signals, as clearly as possible to reflect distribution of underground medium.

The purpose of GPR measurement is to acquire the geological interpretation. According to the waveform and strength characteristics of the reflected wave group, GPR users can infer the geological meanings from the radar image profile and construct geology-geophysical interpretation section through the event tracking. The final radar map of surveyed area provides a basis for engineering design and construction. At some special places, three-dimensional GPR images can provide a more intuitive interpretation ${ }^{[17][18]}$. Owing to the GPR data reflect electrical distribution underground medium, GPR

Natural Science Foundation of China (51409088) 
users need to organically combine the geological information, drilling and GPR image in data interpretation, set up geologygeophysical model about the surveyed area and speculate underground geological structure.

Now the data processing and interpretation of GPR system is the direct application of own post-processing software. These systems include SIR, EKKO, IDS, RAMAC, GEORADAR series etc. But all kinds of software are restricted by the software dongle and expensive. The processing methods are not complete, and GPR users can't modify original algorithms and add some latest processing methods in software. Therefore, the recognition and conversion of GPR original data is the first key step. On the basis of recognition and conversion success, GPR users can add some latest processing methods [19][20] which are not in own post-processing software and achieve the purpose of the final geological explanation.

\section{EXAMPLES}

This research adopts SIR-10H radar system of American GSSI company and RIS radar system of Italian IDS company as examples to illustrate how to identify the original measurement data.

For SIR system, the suffix of original data is the form of *.dzt. The original data is test.dzt, the central frequency of antenna is $400 \mathrm{MHz}$. It is opened in ultra-editor and shown in Fig. 1 and Fig.2.

The GPR original data is saved as the form of hexadecimal numbers. The file header information is displayed on the row of $\mathrm{c} 0 \mathrm{~h}$ in Fig. 1 and the rest is zero. The header file bytes are 1,024(400 h) in Fig.2, the file header and the record are separated from the position of the black and vertical arrow. One data takes two bytes, each record has 512 sampling points, so each trace has 1,024 bytes.

For RIS system, the suffix of original data is the form of *.dt. The original data is LID10001.dt and the central frequency of antenna is $1.6 \mathrm{GHz}$. It is opened in ultra-editor and shown in Fig.3. But it is not the same surveyed area with SIR.

The header file bytes are 15,420 , one data takes two bytes, each trace has 512 sampling points and 1,024 bytes. Furthermore, there is an additional four bytes at the beginning of each trace record. In fact, each trace has 1,028 bytes. The position of the black and vertical arrow is the separate place between the header file and the record file in Fig.3. The part at the back of the black and vertical arrow is belong to trace record file part.

The above results show that GPR original data are saved in the form of the hexadecimal numbers with a header file part. However, for different radar systems, the file header is different and data organization in each trace record is also different.

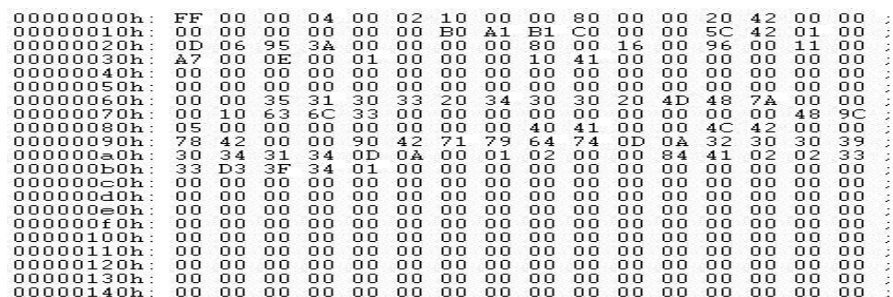

Fig.1. Header file part in original data(SIR)

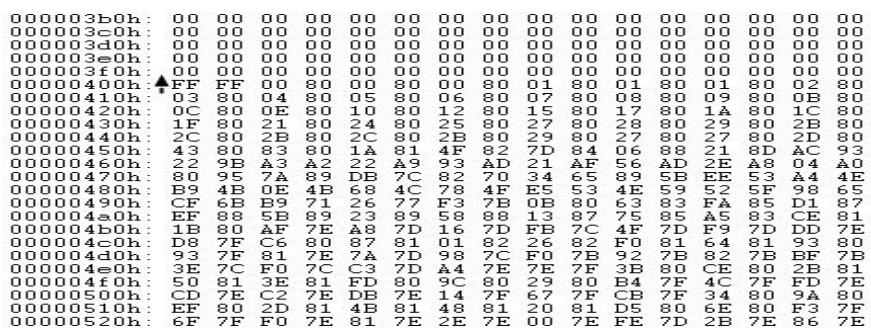

Fig.2. Record file part in original data(SIR)

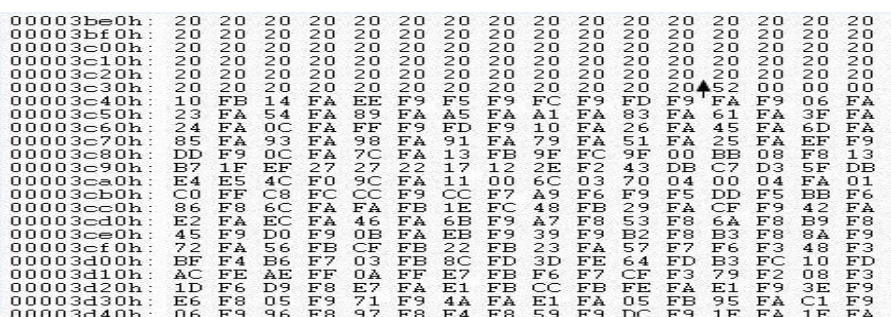

Fig.3. Header file part and record file part in IDS system

Based on the above analysis, this article adopts Visual $\mathrm{C}+$ +6.0 as a development tool to implement the conversion of the GPR original data. The internal hexadecimal data are converted into identifiable decimal data (ASCII data). According to the ASCII data, the waveform are displayed by the following format:

$$
X(i, j)=(A(i, j)-32768) / 32768
$$

Where $i$ is sampling point number, $\mathrm{j}$ is trace number, $A(i, j)$ is ASCII data and $X(i, j)$ is waveform data or the amplitude of wave. The number (32768) is as the waveform zero position.

The detailed conversion process can be displayed through the program flow chart (Fig.4). The executing results of the program are shown below:

For SIR system, the original data file is test.dzt, and the converted file is test.txt (Fig.5). Text data permutation style is the row-priority arrangement. Radar image are displayed in the form of waveform changed area (Fig.6). The horizontal axis represents scan numbers. One trace is a scan or a stack of several scans. The vertical axis stands for sampling points. The Fig.6 is the same with the outcome displayed in the software of Radan developed by GSSI company. 


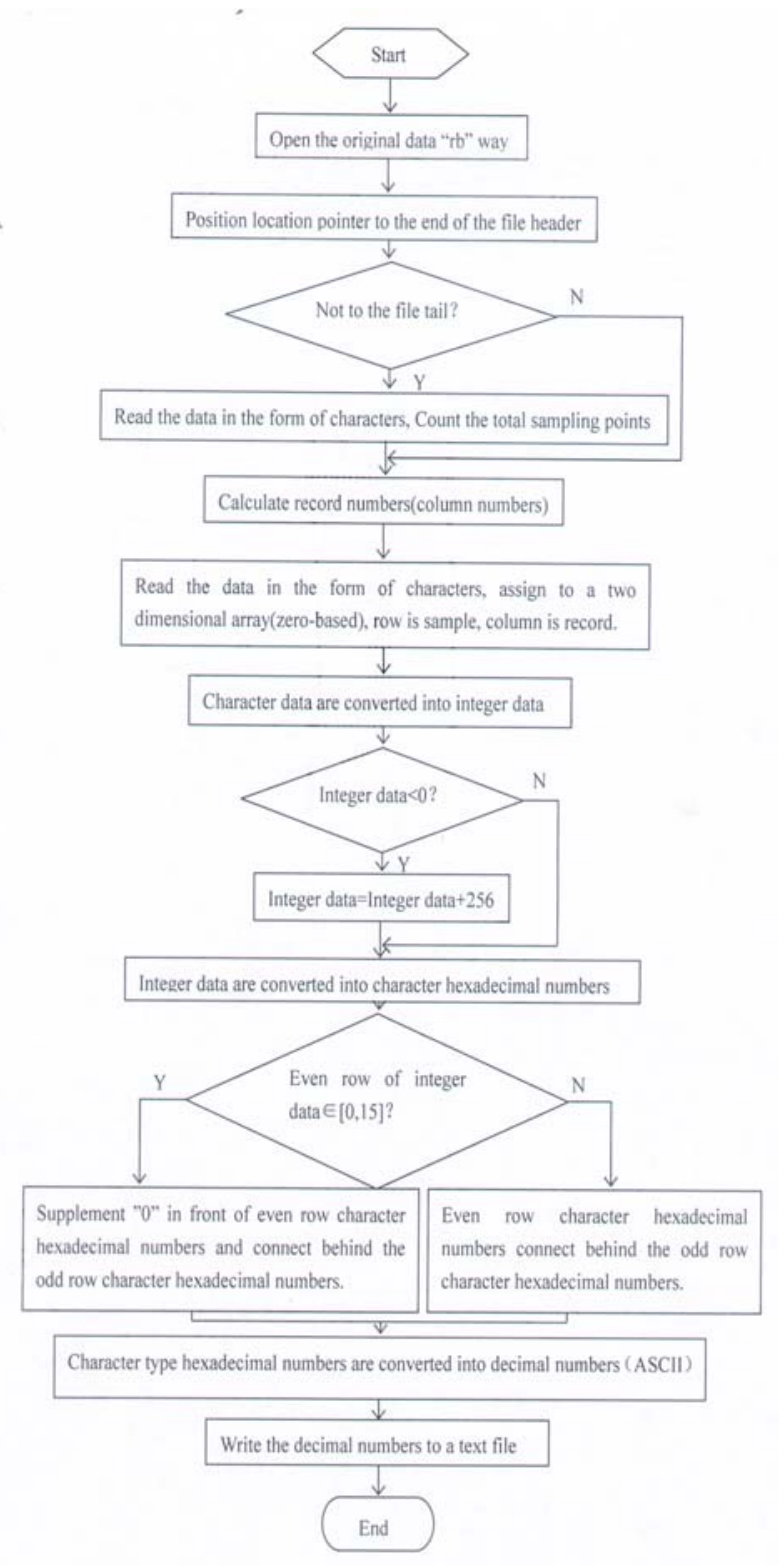

Fig.4. Recognition and conversion program flow chart

For RIS system, the original data file is LID10001.dt, and the converted file is LID10001.txt (Fig.7).

Text data permutation pattern is identical with test.txt. Radar image are displayed in the form of grayscale ${ }^{[21]}$ (Fig.8). The horizontal coordinate denotes scan numbers and the vertical coordinate signifies sampling points. The Fig. 8 is equal to the result displayed in the software of GRED developed by IDS company.

Experiments indicate that the converted image and the original image generated by software in two radar systems is the same. Therefore it proves that the data conversion is correct.

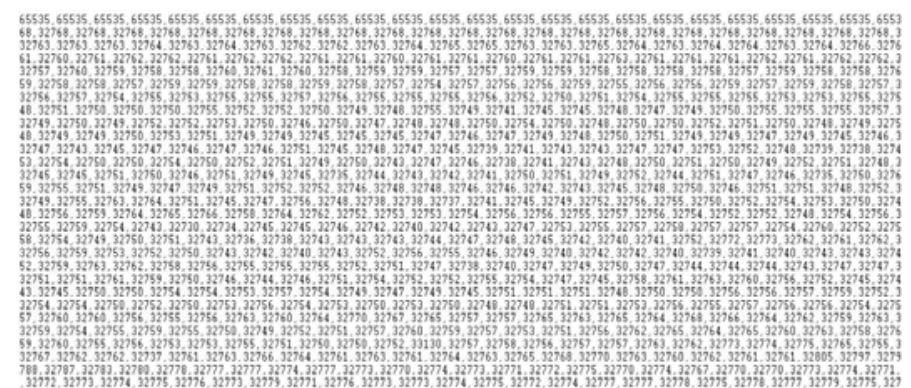

Fig.5. Converted decimal text file (test.txt) (SIR)

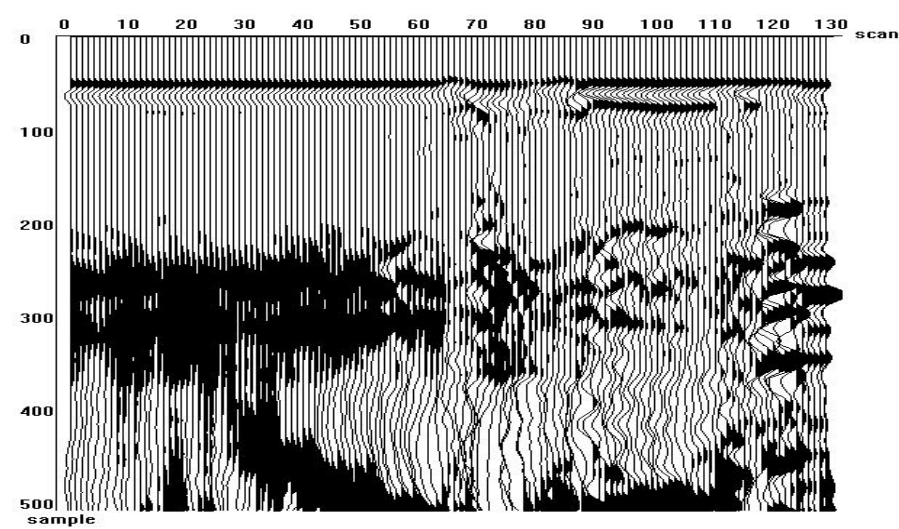

Fig.6. Wiggle figure profile generated by test.txt (SIR)

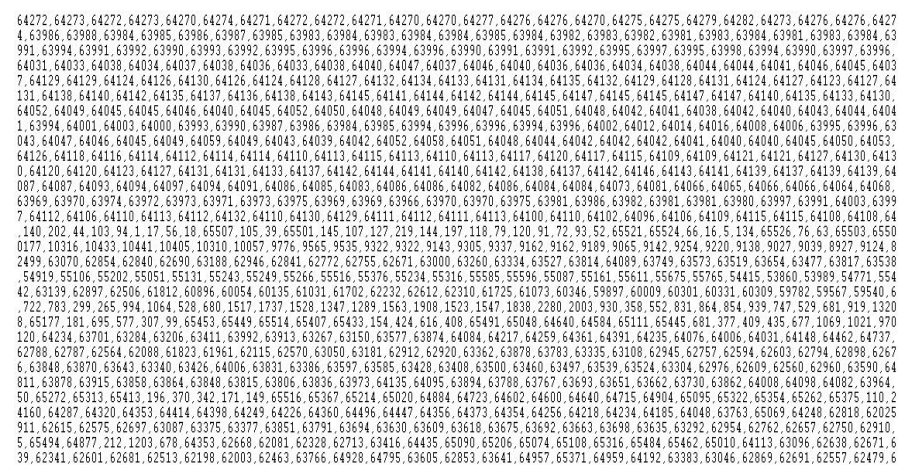

Fig.7. Converted decimal text file (LID10001.txt) (RIS)

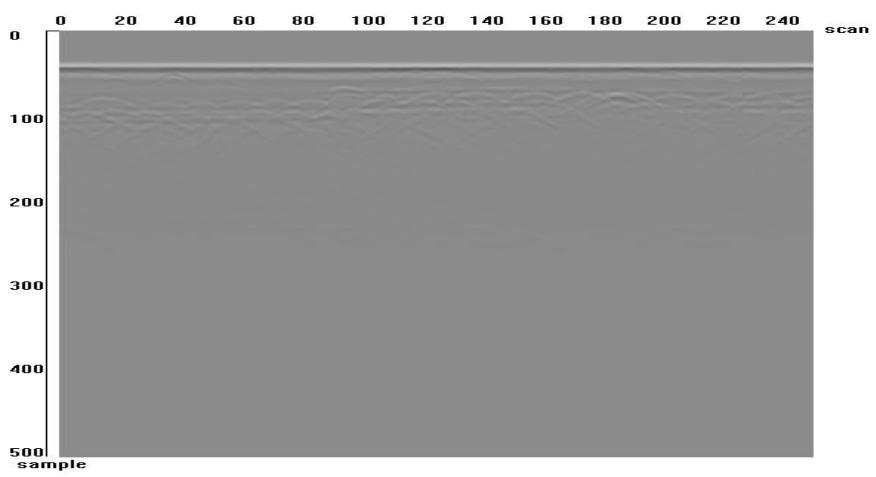

Fig.8. Grayscale image generated by LID10001.txt (RIS)

Furthermore, the conversion program is suitable for most of the radar systems. It will have the widespread application prospect in the future. 


\section{CONCLUSIONS}

We can obtain some conclusions from the above experimental results of the two group radar systems.

- The conversion-program of the GPR field data turns out to be feasible and correct by means of two group different radar systems.

- GPR users can completely eliminate the limit of software dongle and freely add some latest algorithms and data processing methods through the recognition and conversion program. It is very convenient to GPR users.

- This paper also has two aspects of practical significance. One is providing a way to eliminate software dongle restrictions for data processing with flexibility for GPR data processing and radar image analysis. $\mathrm{b}$ providing a basic platform for the secondary development of post-processing software or special post-processing software development.

\section{ACKNOWLEDGMENT}

This research is funded by the Natural Science Foundation of China (51409088). We express our gratitude to harbour laboratory and civil engineering laboratory. Harbour laboratory provides SIR radar system and civil engineering laboratory supplies RIS radar system. We kindly thank Cai Hui and Gao Lei for the fieldwork operations and the geophysical data acquisition.

\section{REFERENCES}

[1] L.M. David and R.J. Puskars, A Subsurface Electromagnetic Pulse Radar, Geophysics, vol. 41(3), 1967, pp. 506-518.

[2] R.N. Bohidar and J.F. Hermance, The GPR Refraction Method, Geophysics, vol. 67(5), 2002, pp. 1474-1485.

[3] I.L. Al-Qadi, Z. Leng, and L. Al, In-place Hot Mix Asphalt Density Estimation Using Ground Penetrating Radar, Ph.D. Thesis, Illinois University, 2011, pp. 28-46.

[4] F.N. Kong and By., Performance of a GPR System Which Uses Step Frequency Signals: J. Appl. Geophys., vol. 33(1-3), 1995, pp. 15-26.
[5] G.F. Stickley, D. Noon, and M. Cherniakov, Gated Stepped-Frequency Ground Penetrating Radar, J. Appl. Geophys., vol. 4(2 4) 2000, pp. 259-269.

[6] L.J. Zhou, R.W. Dong, B. Xu, and Y. Q. Yang, The Application of Ground Penetrating Radar in City Geological Investigation, Chin. J. Eng. Geophys., vol. 6(5), 2009, pp. 632-635.

[7] L.B. Liu and S.A. Arcone, Forward and inverse problems in GPR research, J. Appl. Geophys., vol. 99, 2013, pp. 66-67.

[8] M. Zajc, Ž. Pogaěník, and A. Gosar, Ground penetrating radar and structural geological mapping investigation of karst and tectonic features in flyschoid rocks as geological hazard for exploitation, Int. J. Rock Mech. Min., vol. 67, 2014, pp. 78-87.

[9] A.S. Venkatachalam, X.L. Xu, D. Huston, and T. Xia, Development of a new high speed dual-channel impulse ground penetrating radar, IEEE J. STARS., vol. 7(3), 2014, pp. 753-760.

[10] S. Busch, L. Weihermüller, J.A. Huisman, et al., Coupled hydro geophysical inversion of time-lapse surface GPR data to estimate hydraulic properties of a layered subsurface, Water Resour. Res., vol. 49 2013, pp. 8480-8494.

[11] E. Forte, M. Dossi, R.R. Colucci, and M. Pipan, A new fast methodology to estimate the density of frozen materials by means of common offset GPR data, J. Appl. Geophys., vol. 99, 2013, pp. 135-145.

[12] S. Raffaele, A. Cuccaro, A. Dell'Aversano, and C. Ilaria, Ground clutter removal in GPR surveys, IEEE J. STARS., vol. 7(3), 2014, pp. 792-798.

[13] Q.D. He and W.G. Xiong, Application of Geophysical Tutorial - Seismic Exploration, Geology Publishing House, China, 1990, pp. 130-162.

[14] D.X. Li, Ground Penetrating Radar (GPR) Method and Application, Geology Publishing House, China, 1994, pp. 64-89.

[15] W.C. Yang, Geophysical Inversion \& Seismic Tomography, China Geology Publishing House, 1989.

[16] Y. Yao, Z.B. Zhan, and S.H. Qian, Seismic Exploration New Technologies and New Methods, China University of Geosciences Press, 1991, pp. 121-126.

[17] C.Ç. Yalçiner, Investigation of the subsurface geometry of fissure-ridge travertine with GPR, Pamukkale, western Turkey, J. Geophys. Eng., 10 035001 doi:10.1088/1742-2132/10/3/035001, 2013.

[18] Y.Q. Yang, The application research on three-dimensional GPR image used in grouting effect evaluation of subgrade void, Adv. Mater. Res., vol. 1065-1069, Part 2, 2014, pp. 725-732.

[19] N. Diamanti and A.P. Annan, Characterizing the energy distribution around GPR antennas, J. Appl. Geophys., vol. 99, 2013, pp. 83-90.

[20] X.Y. Xie, H. Qin, C. Yu, and L.B. Liu, An automatic recognition algorithm for GPR images of RC structure voids, J. Appl. Geophys., vol. 99, 2013, pp. 125-134.

[21] B. He, T.Y. Ma, Y.J. Wang, and H.L. Zhu, Visual C++ Digital Image Processing, Posts \& Telecom Press, China, 2001, pp. 92-110. 